\title{
Article
}

\section{De Moivre's and Euler Formulas for Matrices of Hybrid Numbers}

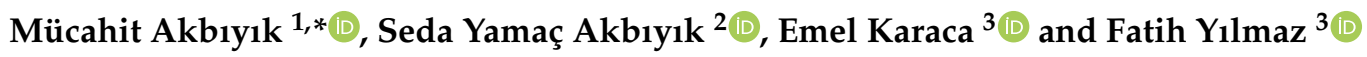 \\ 1 Department of Mathematics, Beykent University, Beykent, Büyükçekmece, Istanbul 34528, Turkey \\ 2 Department of Computer Engineering, Istanbul Gelisim University, Ambarli, Avcllar, Istanbul 34315, Turkey; \\ syamac@gelisim.edu.tr \\ 3 Department of Mathematics, Ankara Hacı Bayram Veli University, Polatlı, Ankara 06900, Turkey; \\ emel.karaca@hbv.edu.tr (E.K.); fatih.yilmaz@hbv.edu.tr (F.Y.) \\ * Correspondence: mucahitakbiyik@beykent.edu.tr
}

Citation: Akbıyık, M.; Yamaç Akbıyık, S.; Karaca, E.; Yılmaz, F. De Moivre's and Euler Formulas for Matrices of Hybrid Numbers. Axioms 2021, 10, 213. https://doi.org/ $10.3390 /$ axioms 10030213

Academic Editor: Ivan Gutman

Received: 13 July 2021

Accepted: 30 August 2021

Published: 6 September 2021

Publisher's Note: MDPI stays neutral with regard to jurisdictional claims in published maps and institutional affiliations.

Copyright: (c) 2021 by the authors. Licensee MDPI, Basel, Switzerland. This article is an open access article distributed under the terms and conditions of the Creative Commons Attribution (CC BY) license (https:// creativecommons.org/licenses/by/ $4.0 /)$.

\begin{abstract}
It is known that the hybrid numbers are generalizations of complex, hyperbolic and dual numbers. Recently, they have attracted the attention of many scientists. At this paper, we provide the Euler's and De Moivre's formulas for the $4 \times 4$ matrices associated with hybrid numbers by using trigonometric identities. Also, we give the roots of the matrices of hybrid numbers. Moreover, we give some illustrative examples to support the main formulas.
\end{abstract}

Keywords: hybrid numbers; matrix representation; Euler and De Moivre's formulas

\section{Introduction}

The complex number systems and their well-known properties have significant roles in algebraic number theory, geometry, dynamic equations, physics, etc. One of the well-known properties is the computation of roots and powers of complex numbers. An alternative way for obtaining roots and powers of complex numbers is to write polar forms for them. Therefore, the theorem, which is called De Moivre's Theorem, for the conversion of complex numbers to their polar forms was introduced by the French mathematician, Abraham De Moivre.

The complicated concepts for powers of complex number, in polar form, could be basically re-expressed by the following formula which is the expression of De Moivre's formula:

$$
(\cos \theta+i \sin \theta)^{n}=(\cos n \theta+i \sin n \theta) .
$$

Considering the De Moivre's formula, Euler's formula, which was introduced by Leonhard Euler, could be exploitted to reduce any trigonometric identity to much easier exponential identities. Hence, Euler's formula is given as follows:

$$
\cos \theta+i \sin \theta=e^{i \theta}
$$

Recently, there has been huge amount of interest to the mentioned formulas whether they could be applied to many special number systems such as quaternion number system, which is the expansion of complex number system. In recent years, many researchers have interested in applications of this formula for quaternions. In [1], these formulas have been generalized for quaternions and also denoted the existence of unaccountably many unit quaternions satisfying $x^{n}=1$. In [2], the authors have studied the Euler's and De Moivre's 
formulas for the matrices associated with quaternions and also obtained any powers of these matrices. That is, for given

$$
A=\left(\begin{array}{cccc}
\cos \theta & -u_{1} \sin \theta & -u_{2} \sin \theta & -u_{3} \sin \theta \\
u_{1} \sin \theta & \cos \theta & -u_{3} \sin \theta & u_{2} \sin \theta \\
u_{2} \sin \theta & -u_{3} \sin \theta & \cos \theta & -u_{1} \sin \theta \\
u_{3} \sin \theta & -u_{2} \sin \theta & u_{1} \sin \theta & \cos \theta
\end{array}\right)
$$

where $q=\cos \theta+u \sin \theta, u=\left(u_{1}, u_{2}, u_{3}\right)$, the $n$th power of this matrix is written as

$$
A^{n}=\left(\begin{array}{cccc}
\cos n \theta & -u_{1} \sin n \theta & -u_{2} \sin n \theta & -u_{3} \sin n \theta \\
u_{1} \sin n \theta & \cos n \theta & -u_{3} \sin n \theta & u_{2} \sin n \theta \\
u_{2} \sin n \theta & -u_{3} \sin n \theta & \cos n \theta & -u_{1} \sin n \theta \\
u_{3} \sin n \theta & -u_{2} \sin n \theta & u_{1} \sin n \theta & \cos n \theta
\end{array}\right) .
$$

In [3], these formulas for the quaternions with the dual number coefficients are obtained. Furthermore, solutions of the equation $x^{n}=1$ is discussed whether they have solutions for a general unit dual quaternion. Moreover, these formulas have been studied for split quaternions, which were introduced by J. Cockle. In [4], De Moivre's formula for split quaternions and roots of a split quaternion using this formula have been expressed. In [5], the authors have studied De Moivre's formula for real matrices of timelike and spacelike split quaternions, respectively. Additionally, the Euler theorem for real matrices of pure split quaternions have been mentioned in the same study. Also, in [6], these formulas have been examined by using matrix theory over the split quaternions. In [7], the authors consider the unit dual quaternion and obtain the De Moivre's formula. In [8], the Euler and De Moivre's formulas for fundamental matrices of commutative quaternions have been obtained. In [9], the author obtained the De Moivre's formula of the hyperbolic quaternions. In [10], the authors studied two generalizations of dual-hyperbolic balancing numbers: dual-hyperbolic Horadam numbers and dual-hyperbolic k-balancing numbers. They gave Catalan's identity, Cassini's identity, and d'Ocagne's identity for them. Moreover, in [11], the authors introduced a special kind of spacelike hybrid number, namely the $\mathrm{F}(\mathrm{p}, \mathrm{n})$ Fibonacci hybrid numbers and they gave some of their properties. Also, in [12], the author studied on a representation of De Moivre's formula of the Pauli quaternions. Recently, there has been huge amount of papers deal with the geometric and physical applications of complex, hyperbolic and dual numbers which are well-known two dimensional number systems. At this content, Ozdemir, [13], introduced the hybrid numbers as below:

$$
\mathbb{H}=\left\{Z=a+b i+c \varepsilon+d h \mid a, b, c, d \in \mathbb{R}, i^{2}=-1, \varepsilon^{2}=0, h^{2}=1\right\} .
$$

In [14], the authors studied the concept of similarity for hybrid numbers by using the solutions of some linear equations. In [15], the author gave a new method for finding $n t h$ roots of a $2 \times 2$ real matrix with the help of hybrid numbers. He gave the De Moivre's formula according to type and character of the $2 \times 2$ real matrix.

\section{Matrix Representations for Any Unit Hybrid Number}

In this section, we give some fundamental definitions and properties. Let us denote any hybrid number as $Z=a+b i+c \varepsilon+d h \in \mathbb{H}$. Here, the real number $a$ is called the scalar part, denoted by $S(Z)$ and $b i+c \varepsilon+d h$ is also called the vector part, denoted by $V(Z)$ in [13].

Definition 1. The conjugate of a hybrid number $Z$ is

$$
\bar{Z}=S(Z)-V(Z)=a-b i-c \varepsilon-d h .
$$


Moreover, by using hybridian product, we have $\mathrm{ZZ}=\bar{Z} Z$. Additionally,

$$
C(Z)=Z \bar{Z}=\bar{Z} Z=a^{2}+(b-c)^{2}-c^{2}-d^{2}
$$

is called the character of the hybrid number of Z, [13].

Definition 2. The hybrid number $Z$ is called spacelike, timelike or lightlike if $C(Z)<0, C(Z)>0$ or $C(Z)=0$, respectively. The characters of the hybrid number $Z$ are identified by exploiting these conditions. Furthermore, $\sqrt{|C(Z)|}$ represents the norm of the hybrid number $Z$, denoted by ||Z|| [13].

Definition 3. For the hybrid number $Z=a+b i+c \varepsilon+d h$, the vector, $\varepsilon_{Z}=((b-c), c, d)$ is called the hybrid vector of the number of $Z$ and it can be written as [13].

$$
C_{\varepsilon}(Z)=-(b-c)^{2}+c^{2}+d^{2}
$$

Therefore, the hybrid number $Z$ is called elliptic, hyperbolic or parabolic, if $C_{\varepsilon}(Z)<0, C_{\varepsilon}(Z)>0$ or $C_{\varepsilon}(Z)=0$, respectively. The types of the hybrid number $Z$ are identified by using these conditions. Furthermore, $\sqrt{\left|C_{\varepsilon}(Z)\right|}$ is the norm of the hybrid vector of $Z$, denoted by $N(Z)$ [13].

The polar representation of a hybrid number depends on the type of the hybrid vector and the casual character. Taking into account these conditions, we summarized the polar representations of the hybrid numbers in the following Table 1 with data from [13] :

\begin{tabular}{|c|c|c|}
\hline Character of Hybrid Number & Polar Form & Conditions \\
\hline Unit elliptic hybrid & $Z=\cos \theta+V \sin \theta$ & $\begin{array}{c}V=\frac{b i+c \mathcal{c}+d h}{N(Z)} \\
\cos \theta=\frac{a}{\|Z\|} \\
\sin \theta=\frac{N(Z)}{\|Z\|} \\
V^{2}=-1\end{array}$ \\
\hline Unit spacelike hyperbolic hybrid & $Z=\sinh \theta+V \cos h \theta$ & $\begin{array}{c}V=\frac{b i+c \mathcal{c}+d h}{N(Z)} \\
\cosh \theta=\frac{N(Z)}{\|Z\|} \\
\sinh \theta=\frac{a}{\|Z\|} \\
V^{2}=1\end{array}$ \\
\hline Unit parabolic hybrid & $Z=\epsilon+V$ & $\begin{array}{c}V=\frac{b i+c \varepsilon+d h}{N(Z)} \\
\epsilon=\operatorname{sgn}(S(Z)) \\
V^{2}=0\end{array}$ \\
\hline Unit lightlike hyperbolic hybrid & $Z=a(1+V)$ & $\begin{array}{c}V=\frac{b i+c \mathcal{E}+d h}{N(Z)} \\
V^{2}=1\end{array}$ \\
\hline Unit timelike hyperbolic hybrid & $Z= \pm(\cosh \theta+V \sinh \theta)$ & $\begin{array}{c}V=\frac{b i+c \varepsilon+d h}{N(Z)} \\
\cosh \theta=\frac{a}{\|Z\|} \\
\sinh \theta=\frac{N(Z)}{\|Z\|} \\
V^{2}=1\end{array}$ \\
\hline
\end{tabular}

Table 1. The polar representations of the hybrid numbers.

Moreover, the unit parabolic hybrid number also can be rewritten as

$$
Z=\cos _{g} \theta+V \sin _{g} \theta
$$


if $Z$ is a unit parabolic hybrid number and $c \neq 0, V=\frac{b i+c \varepsilon+d h}{c}, \cos _{g} \theta=a, \sin _{g} \theta=c$, $\mathbf{V}^{2}=0$. Here $\cos _{g} \theta$ and $\sin _{g} \theta$ denote the cosine and sine Galilean trigonometric functions, respectively. In [14], the left matrix representation of the hybrid number $Z$ is given by

$$
\mathbf{Z}_{L}=\left[\begin{array}{cccc}
a & c-b & b & d \\
b & a-d & 0 & b \\
c & -d & a+d & b-c \\
d & c & -b & a
\end{array}\right]
$$

Similarly, the right representation of the hybrid number $Z$ is given by

$$
\mathbf{Z}_{R}=\left[\begin{array}{cccc}
a & c-b & b & d \\
b & a+d & 0 & -b \\
c & d & a-d & c-b \\
d & -c & b & a
\end{array}\right]
$$

\section{De Moivre's Formula of $4 \times 4$ Real Matrices of Hybrid Numbers}

In this section, we examine both the left and right matrix representations for each conditions. We give all the investigation for

$$
\mathbf{Z}=x+\mathbf{V} y=x+\left(v_{1}, v_{2}, v_{3}\right) y \in \mathbb{H}
$$

as below:

I. If $Z$ is a unit elliptic hybrid number, then

$$
\mathbf{Z}_{L}=\left[\begin{array}{cccc}
\cos \theta & \left(v_{2}-v_{1}\right) \sin \theta & v_{1} \sin \theta & v_{3} \sin \theta \\
v_{1} \sin \theta & \cos \theta-v_{3} \sin \theta & 0 & v_{1} \sin \theta \\
v_{2} \sin \theta & -v_{3} \sin \theta & \cos \theta+v_{3} \sin \theta & \left(v_{1}-v_{2}\right) \sin \theta \\
v_{3} \sin \theta & v_{2} \sin \theta & -v_{1} \sin \theta & \cos \theta
\end{array}\right] .
$$

If $Z$ is a spacelike unit hyperbolic hybrid number,

$$
\mathbf{Z}_{L}=\left[\begin{array}{cccc}
\sinh \theta & \left(v_{2}-v_{1}\right) \cosh \theta & v_{1} \cosh \theta & v_{3} \cosh \theta \\
v_{1} \cosh \theta & \sinh \theta-v_{3} \cosh \theta & 0 & v_{1} \cosh \theta \\
v_{2} \cosh \theta & -v_{3} \cosh \theta & \sinh \theta+v_{3} \cosh \theta & \left(v_{1}-v_{2}\right) \cosh \theta \\
v_{3} \cosh \theta & v_{2} \cosh \theta & -v_{1} \cosh \theta & \sinh \theta
\end{array}\right] .
$$

If $Z$ is a timelike unit hyperbolic hybrid number,

$$
\mathbf{Z}_{L}= \pm\left[\begin{array}{cccc}
\cosh \theta & \left(v_{2}-v_{1}\right) \sinh \theta & v_{1} \sinh \theta & v_{3} \sinh \theta \\
v_{1} \sinh \theta & \cosh \theta-v_{3} \sinh \theta & 0 & v_{1} \sinh \theta \\
v_{2} \sinh \theta & -v_{3} \sinh \theta & \cosh \theta+v_{3} \sinh \theta & \left(v_{1}-v_{2}\right) \sinh \theta \\
v_{3} \sinh \theta & v_{2} \sinh \theta & -v_{1} \sinh \theta & \cosh \theta
\end{array}\right]
$$

If $Z$ is a unit parabolic hybrid number, then we get

$$
\mathbf{Z}_{L}=\left[\begin{array}{cccc}
\epsilon & \left(v_{2}-v_{1}\right) & v_{1} & v_{3} \\
v_{1} & \epsilon-v_{3} & 0 & v_{1} \\
v_{2} & -v_{3} & \epsilon+v_{3} & \left(v_{1}-v_{2}\right) \\
v_{3} & v_{2} & -v_{1} & \epsilon
\end{array}\right]
$$


Moreover, if $Z$ is a unit parabolic hybrid number and especially $c \neq 0$, then

$$
\mathbf{Z}_{L}=\left[\begin{array}{cccc}
\cos _{g} \theta & \left(v_{2}-v_{1}\right) \sin _{g} \theta & v_{1} \sin _{g} \theta & v_{3} \sin _{g} \theta \\
v_{1} \sin _{g} \theta & \cos _{g} \theta-v_{3} \sin \theta & 0 & v_{1} \sin _{g} \theta \\
v_{2} \theta & -v_{3} \theta & \cos _{g} \theta+v_{3} \sin \theta & \left(v_{1}-v_{2}\right) \sin _{g} \theta \\
v_{3} \sin _{g} \theta & v_{2} \sin _{g} \theta & -v_{1} \sin _{g} \theta & \cos _{g} \theta
\end{array}\right]
$$

If $Z$ is a lightlike hybrid number, then

$$
\mathbf{Z}_{L}=\left[\begin{array}{cccc}
a & a\left(v_{2}-v_{1}\right) & a v_{1} & a v_{3} \\
a v_{1} & a-a v_{3} & 0 & v_{1} \\
a v_{2} & -a v_{3} & a+a v_{3} & a\left(v_{1}-v_{2}\right) \\
a v_{3} & a v_{2} & -a v_{1} & a
\end{array}\right]
$$

II. If $Z$ is a unit elliptic hybrid number,

$$
\mathbf{Z}_{R}=\left[\begin{array}{cccc}
\cos \theta & \left(v_{2}-v_{1}\right) \sin \theta & v_{1} \sin \theta & v_{3} \sin \theta \\
v_{1} \sin \theta & \cos \theta+v_{3} \sin \theta & 0 & -v_{1} \sin \theta \\
v_{2} \sin \theta & v_{3} \sin \theta & \cos \theta-v_{3} \sin \theta & \left(v_{2}-v_{1}\right) \sin \theta \\
v_{3} \sin \theta & -v_{2} \sin \theta & v_{1} \sin \theta & \cos \theta
\end{array}\right]
$$

If $Z$ is a spacelike unit hyperbolic hybrid number,

$$
\mathbf{Z}_{R}=\left[\begin{array}{cccc}
\sinh \theta & \left(v_{2}-v_{1}\right) \cosh \theta & v_{1} \cosh \theta & v_{3} \cosh \theta \\
v_{1} \cosh \theta & \sinh \theta+v_{3} \cosh \theta & 0 & -v_{1} \cosh \theta \\
v_{2} \cosh \theta & v_{3} \cosh \theta & \sinh \theta-v_{3} \cosh \theta & \left(v_{2}-v_{1}\right) \cosh \theta \\
v_{3} \cosh \theta & -v_{2} \cosh \theta & v_{1} \cosh \theta & \sinh \theta
\end{array}\right] .
$$

If $Z$ is a timelike unit hyperbolic hybrid number,

$$
\mathbf{Z}_{R}= \pm\left[\begin{array}{cccc}
\cosh \theta & \left(v_{2}-v_{1}\right) \sinh \theta & v_{1} \sinh \theta & v_{3} \sinh \theta \\
v_{1} \sinh \theta & \cosh \theta+v_{3} \sinh \theta & 0 & -v_{1} \sinh \theta \\
v_{2} \sinh \theta & v_{3} \sinh \theta & \cosh \theta-v_{3} \sinh \theta & \left(v_{2}-v_{1}\right) \sinh \theta \\
v_{3} \sinh \theta & -v_{2} \sinh \theta & v_{1} \sinh \theta & \cosh \theta
\end{array}\right]
$$

If $Z$ is a unit parabolic hybrid number,

$$
\mathbf{Z}_{R}=\left[\begin{array}{cccc}
\epsilon & \left(v_{2}-v_{1}\right) & v_{1} & v_{3} \\
v_{1} & \epsilon+v_{3} & 0 & -v_{1} \\
v_{2} & v_{3} & \epsilon-v_{3} & \left(v_{2}-v_{1}\right) \\
v_{3} & -v_{2} & v_{1} & \epsilon
\end{array}\right]
$$

Furthermore, if $Z$ is a unit parabolic hybrid number and especially $c \neq 0$, then

$$
\mathbf{Z}_{R}=\left[\begin{array}{cccc}
\cos _{g} \theta & \left(v_{2}-v_{1}\right) \sin \theta & v_{1} \sin _{g} \theta & v_{3} \sin _{g} \theta \\
v_{1} \sin _{g} \theta & \cos _{g} \theta+v_{3} \sin _{g} \theta & 0 & -v_{1} \sin _{g} \theta \\
v_{2} \sin _{g} \theta & v_{3} \sin _{g} \theta & \cos \theta-v_{3} \sin \theta & \left(v_{2}-v_{1}\right) \sin _{g} \theta \\
v_{3} \sin _{g} \theta & -v_{2} \sin _{g} \theta & v_{1} \sin _{g} \theta & \cos _{g} \theta
\end{array}\right] .
$$

If $Z$ is a lightlike hybrid number,

$$
\mathbf{Z}_{R}=\left[\begin{array}{cccc}
a & a\left(v_{2}-v_{1}\right) & a v_{1} & a v_{3} \\
a v_{1} & a+a v_{3} & 0 & -a v_{1} \\
a v_{2} & a v_{3} & a-v_{3} & a\left(v_{2}-v_{1}\right) \\
a v_{3} & -a v_{2} & a v_{1} & a
\end{array}\right]
$$


Here, we compute the right matrix multiplications for given four conditions at the following four lemmas.

Lemma 1. Given two matrices

$$
\mathbf{Z}_{R}=\left[\begin{array}{cccc}
\cos \theta & \left(v_{2}-v_{1}\right) \sin \theta & v_{1} \sin \theta & v_{3} \sin \theta \\
v_{1} \sin \theta & \cos \theta+v_{3} \sin \theta & 0 & -v_{1} \sin \theta \\
v_{2} \sin \theta & v_{3} \sin \theta & \cos \theta-v_{3} \sin \theta & \left(v_{2}-v_{1}\right) \sin \theta \\
v_{3} \sin \theta & -v_{2} \sin \theta & v_{1} \sin \theta & \cos \theta
\end{array}\right]
$$

and

$$
\boldsymbol{W}_{R}=\left[\begin{array}{cccc}
\cos \alpha & \left(v_{2}-v_{1}\right) \sin \alpha & v_{1} \sin \alpha & v_{3} \sin \alpha \\
v_{1} \sin \alpha & \cos \alpha+v_{3} \sin \alpha & 0 & -v_{1} \sin \alpha \\
v_{2} \sin \alpha & v_{3} \sin \alpha & \cos \alpha-v_{3} \sin \alpha & \left(v_{2}-v_{1}\right) \sin \alpha \\
v_{3} \sin \alpha & -v_{2} \sin \alpha & v_{1} \sin \alpha & \cos \alpha
\end{array}\right]
$$

Then,

$$
\boldsymbol{Z}_{R} \boldsymbol{W}_{R}=\left[\begin{array}{cccc}
\cos (\theta+\alpha) & \left(v_{2}-v_{1}\right) \sin (\theta+\alpha) & v_{1} \sin (\theta+\alpha) & v_{3} \sin (\theta+\alpha) \\
v_{1} \sin (\theta+\alpha) & \cos (\theta+\alpha)+v_{3} \sin (\theta+\alpha) & 0 & -v_{1} \sin (\theta+\alpha) \\
v_{2} \sin (\theta+\alpha) & v_{3} \sin (\theta+\alpha) & \cos (\theta+\alpha)-v_{3} \sin \theta & \left(v_{2}-v_{1}\right) \sin (\theta+\alpha) \\
v_{3} \sin (\theta+\alpha) & -v_{2} \sin (\theta+\alpha) & v_{1} \sin (\theta+\alpha) & \cos (\theta+\alpha)
\end{array}\right] .
$$

Proof. It can be seen easily by matrix multiplication and using the following trigonometric equalities:

$$
\begin{gathered}
\cos (\theta+\alpha)=\cos \theta \cos \alpha-\sin \theta \sin \theta, \\
\sin (\theta+\alpha)=\sin \theta \cos \alpha+\cos \theta \sin \theta, \\
v_{3}^{2}-v_{1}^{2}+2 v_{1} v_{2}=-1 .
\end{gathered}
$$

Lemma 2. Let

$$
\mathbf{Z}_{R}=\left[\begin{array}{cccc}
\sinh \theta & \left(v_{2}-v_{1}\right) \cosh \theta & v_{1} \cosh \theta & v_{3} \cosh \theta \\
v_{1} \cosh \theta & \sinh \theta+v_{3} \cosh \theta & 0 & -v_{1} \cosh \theta \\
v_{2} \cosh \theta & v_{3} \cosh \theta & \sinh \theta-v_{3} \cosh \theta & \left(v_{2}-v_{1}\right) \cosh \theta \\
v_{3} \cosh \theta & -v_{2} \cosh \theta & v_{1} \cosh \theta & \sinh \theta
\end{array}\right]
$$

and

$$
\boldsymbol{W}_{R}=\left[\begin{array}{cccc}
\sinh \alpha & \left(v_{2}-v_{1}\right) \cosh \alpha & v_{1} \cosh \alpha & v_{3} \cosh \alpha \\
v_{1} \cosh \alpha & \sinh \alpha+v_{3} \cosh \alpha & 0 & -v_{1} \cosh \alpha \\
v_{2} \cosh \alpha & v_{3} \cosh \alpha & \sinh \alpha-v_{3} \cosh \alpha & \left(v_{2}-v_{1}\right) \cosh \alpha \\
v_{3} \cosh \alpha & -v_{2} \cosh \alpha & v_{1} \cosh \alpha & \sinh \alpha
\end{array}\right]
$$

Then,

$$
\boldsymbol{Z}_{R} \boldsymbol{W}_{R}=\left[\begin{array}{cccc}
\cosh (\theta+\alpha) & \left(v_{2}-v_{1}\right) \sinh (\theta+\alpha) & v_{1} \sinh (\theta+\alpha) & v_{3} \sinh (\theta+\alpha) \\
v_{1} \sinh (\theta+\alpha) & \cosh (\theta+\alpha)+v_{3} \sinh (\theta+\alpha) & 0 & -v_{1} \sinh (\theta+\alpha) \\
v_{2} \sinh (\theta+\alpha) & v_{3} \sinh (\theta+\alpha) & \cosh (\theta+\alpha)-v_{3} \sinh \theta & \left(v_{2}-v_{1}\right) \sinh (\theta+\alpha) \\
v_{3} \sinh (\theta+\alpha) & -v_{2} \sinh (\theta+\alpha) & v_{1} \sinh (\theta+\alpha) & \cosh (\theta+\alpha)
\end{array}\right]
$$

Proof. It can be seen easily by matrix multiplication and using the following trigonometric equalities:

$$
\begin{gathered}
\cosh (\theta+\alpha)=\cosh \theta \cosh \alpha+\sinh \theta \sinh \theta \\
\sinh (\theta+\alpha)=\sinh \theta \cosh \alpha+\cosh \theta \sinh \theta, \\
v_{3}^{2}-v_{1}^{2}+2 v_{1} v_{2}=1 .
\end{gathered}
$$


Lemma 3. Let

$$
\mathbf{Z}_{R}= \pm\left[\begin{array}{cccc}
\cosh \theta & \left(v_{2}-v_{1}\right) \sinh \theta & v_{1} \sinh \theta & v_{3} \sinh \theta \\
v_{1} \sinh \theta & \cosh \theta+v_{3} \sinh \theta & 0 & -v_{1} \sinh \theta \\
v_{2} \sinh \theta & v_{3} \sinh \theta & \cosh \theta-v_{3} \sinh \theta & \left(v_{2}-v_{1}\right) \sinh \theta \\
v_{3} \sinh \theta & -v_{2} \sinh \theta & v_{1} \sinh \theta & \cosh \theta
\end{array}\right]
$$

and

$$
\boldsymbol{W}_{R}= \pm\left[\begin{array}{cccc}
\cosh \alpha & \left(v_{2}-v_{1}\right) \sinh \alpha & v_{1} \sinh \alpha & v_{3} \sinh \alpha \\
v_{1} \sinh \alpha & \cosh \alpha+v_{3} \sinh \alpha & 0 & -v_{1} \sinh \alpha \\
v_{2} \sinh \alpha & v_{3} \sinh \alpha & \cosh \alpha-v_{3} \sinh \alpha & \left(v_{2}-v_{1}\right) \sinh \alpha \\
v_{3} \sinh \alpha & -v_{2} \sinh \alpha & v_{1} \sinh \alpha & \cosh \alpha
\end{array}\right]
$$

Then,

$$
\boldsymbol{Z}_{R} \boldsymbol{W}_{R}= \pm\left[\begin{array}{cccc}
\cosh (\theta+\alpha) & \left(v_{2}-v_{1}\right) \sinh (\theta+\alpha) & v_{1} \sinh (\theta+\alpha) & v_{3} \sinh (\theta+\alpha) \\
v_{1} \sinh (\theta+\alpha) & \cosh (\theta+\alpha)+v_{3} \sinh (\theta+\alpha) & 0 & -v_{1} \sinh (\theta+\alpha) \\
v_{2} \sinh (\theta+\alpha) & v_{3} \sinh (\theta+\alpha) & \cosh (\theta+\alpha)-v_{3} \sinh \theta & \left(v_{2}-v_{1}\right) \sinh (\theta+\alpha) \\
v_{3} \sinh (\theta+\alpha) & -v_{2} \sinh (\theta+\alpha) & v_{1} \sinh (\theta+\alpha) & \cosh (\theta+\alpha)
\end{array}\right]
$$

Proof. Going on with the similar way at the proofs given above and using the following trigonometric equalities:

$$
\begin{gathered}
\cosh (\theta+\alpha)=\cosh \theta \cosh \alpha+\sinh \theta \sinh \theta \\
\sinh (\theta+\alpha)=\sinh \theta \cosh \alpha+\cosh \theta \sinh \theta \\
v_{3}^{2}-v_{1}^{2}+2 v_{1} v_{2}=1 .
\end{gathered}
$$

The proof can be verified, clearly.

Lemma 4. Let

$$
\mathbf{Z}_{R}=\left[\begin{array}{cccc}
\cos _{g} \theta & \left(v_{2}-v_{1}\right) \sin _{g} \theta & v_{1} \sin _{g} \theta & v_{3} \sin _{g} \theta \\
v_{1} \sin _{g} \theta & \cos _{g} \theta+v_{3} \sin \theta & 0 & -v_{1} \sin \theta \\
v_{2} \sin _{g} \theta & v_{3} \sin _{g} \theta & \cos \theta-v_{3} \sin \theta & \left(v_{2}-v_{1}\right) \sin _{g} \theta \\
v_{3} \sin _{g} \theta & -v_{2} \sin \theta & v_{1} \sin _{g} \theta & \cos \theta
\end{array}\right]
$$

and

$$
\boldsymbol{W}_{R}=\left[\begin{array}{cccc}
\cos _{g} \alpha & \left(v_{2}-v_{1}\right) \sin _{g} \alpha & v_{1} \sin _{g} \alpha & v_{3} \sin \alpha \\
v_{1} \sin _{g} \alpha & \cos \alpha+v_{3} \sin _{g} \alpha & 0 & -v_{1} \sin _{g} \alpha \\
v_{2} \sin _{g} \alpha & v_{3} \sin _{g} \alpha & \cos \alpha-v_{3} \sin \alpha & \left(v_{2}-v_{1}\right) \sin _{g} \alpha \\
v_{3} \sin _{g} \alpha & -v_{2} \sin _{g} \alpha & v_{1} \sin _{g} \alpha & \cos g
\end{array}\right]
$$

Then,

$$
\boldsymbol{Z}_{R} \boldsymbol{W}_{R}=\left[\begin{array}{cccc}
\cos _{g}(\theta+\alpha) & \left(v_{2}-v_{1}\right) \sin _{g}(\theta+\alpha) & v_{1} \sin _{g}(\theta+\alpha) & v_{3} \sin _{g}(\theta+\alpha) \\
v_{1} \sin _{g}(\theta+\alpha) & \cos _{g}(\theta+\alpha)+v_{3} \sin _{g}(\theta+\alpha) & 0 & -v_{1} \sin _{g}(\theta+\alpha) \\
v_{2} \sin _{g}(\theta+\alpha) & v_{3} \sin _{g}(\theta+\alpha) & \cos _{g}(\theta+\alpha)-v_{3} \sin n_{g} \theta & \left(v_{2}-v_{1}\right) \sin _{g}(\theta+\alpha) \\
v_{3} \sin _{g}(\theta+\alpha) & -v_{2} \sin _{g}(\theta+\alpha) & v_{1} \sin _{g}(\theta+\alpha) & \cos _{g}(\theta+\alpha)
\end{array}\right] .
$$

Proof. By following similar steps at the proofs given above and using the following trigonometric equalities:

$$
\begin{gathered}
\cos _{g}(\theta+\alpha)=\cos _{g} \theta \cos _{g} \alpha, \\
\sin _{g}(\theta+\alpha)=\sin _{g} \theta \cos _{g} \alpha+\cos _{g} \theta \sin _{g} \theta, \\
v_{3}^{2}-v_{1}^{2}+2 v_{1} v_{2}=0 .
\end{gathered}
$$

The proof can be seen easily. 
Theorem 1. Let

$$
\mathbf{Z}_{R}=\left[\begin{array}{cccc}
\cos \theta & \left(v_{2}-v_{1}\right) \sin \theta & v_{1} \sin \theta & v_{3} \sin \theta \\
v_{1} \sin \theta & \cos \theta+v_{3} \sin \theta & 0 & -v_{1} \sin \theta \\
v_{2} \sin \theta & v_{3} \sin \theta & \cos \theta-v_{3} \sin \theta & \left(v_{2}-v_{1}\right) \sin \theta \\
v_{3} \sin \theta & -v_{2} \sin \theta & v_{1} \sin \theta & \cos \theta
\end{array}\right]
$$

be a right matrix representation for a unit elliptic hybrid number. Then the nth power of the matrix is given as follows:

$$
Z_{R}^{n}=\left[\begin{array}{cccc}
\cos n \theta & \left(v_{2}-v_{1}\right) \sin n \theta & v_{1} \sin n \theta & v_{3} \sin n \theta \\
v_{1} \sin n \theta & \cos n \theta+v_{3} \sin n \theta & 0 & -v_{1} \sin n \theta \\
v_{2} \sin n \theta & v_{3} \sin n \theta & \cos n \theta-v_{3} \sin n \theta & \left(v_{2}-v_{1}\right) \sin n \theta \\
v_{3} \sin n \theta & -v_{2} \sin n \theta & v_{1} \sin n \theta & \cos n \theta
\end{array}\right] .
$$

Proof. We use the mathematical induction method for all non negative integers $n$. For $n=2$, by applying matrix multiplication:

$$
\mathbf{Z}_{R}^{2}=\left[\begin{array}{cccc}
\cos 2 \theta & \left(v_{2}-v_{1}\right) \sin 2 \theta & v_{1} \sin 2 \theta & v_{3} \sin 2 \theta \\
v_{1} \sin 2 \theta & \cos 2 \theta+v_{3} \sin 2 \theta & 0 & -v_{1} \sin 2 \theta \\
v_{2} \sin 2 \theta & v_{3} \sin 2 \theta & \cos n \theta-v_{3} \sin 2 \theta & \left(v_{2}-v_{1}\right) \sin 2 \theta \\
v_{3} \sin 2 \theta & -v_{2} \sin 2 \theta & v_{1} \sin 2 \theta & \cos 2 \theta
\end{array}\right] .
$$

Suppose that the theorem is true for $n=k$, i.e.:

$$
\mathbf{Z}_{R}^{k}=\left[\begin{array}{cccc}
\cos k \theta & \left(v_{2}-v_{1}\right) \sin k \theta & v_{1} \sin k \theta & v_{3} \sin k \theta \\
v_{1} \sin k \theta & \cos k \theta+v_{3} \sin k \theta & 0 & -v_{1} \sin k \theta \\
v_{2} \sin k \theta & v_{3} \sin k \theta & \cos k \theta-v_{3} \sin k \theta & \left(v_{2}-v_{1}\right) \sin k \theta \\
v_{3} \sin k \theta & -v_{2} \sin k \theta & v_{1} \sin k \theta & \cos k \theta
\end{array}\right] .
$$

Then, let us show that the theorem is valid for $n=k+1$. One can verify that it holds for $n=k+1$ as below:

$$
\mathbf{Z}_{R}^{(k+1)}=\left[\begin{array}{cccc}
\cos (k+1) \theta & \left(v_{2}-v_{1}\right) \sin (k+1) \theta & v_{1} \sin (k+1) \theta & v_{3} \sin (k+1) \theta \\
v_{1} \sin (k+1) \theta & \cos (k+1) \theta+v_{3} \sin (k+1) \theta & 0 & -v_{1} \sin (k+1) \theta \\
v_{2} \sin (k+1) \theta & v_{3} \sin (k+1) \theta & \cos (k+1) \theta-v_{3} \sin (k+1) \theta & \left(v_{2}-v_{1}\right) \sin (k+1) \theta \\
v_{3} \sin (k+1) \theta & -v_{2} \sin (k+1) \theta & v_{1} \sin (k+1) \theta & \cos (k+1) \theta
\end{array}\right] .
$$

Also, we give De Moivre formula for negative powers by following the same way. In other words, for $n=-1$, we obtain the inverse matrix as following:

$$
\begin{aligned}
\mathbf{Z}_{R}^{-1}= & {\left[\begin{array}{cccc}
\cos \theta & -\left(v_{2}-v_{1}\right) \sin \theta & -v_{1} \sin \theta & -v_{3} \sin \theta \\
-v_{1} \sin \theta & \cos \theta-v_{3} \sin \theta & 0 & v_{1} \sin \theta \\
-v_{2} \sin \theta & -v_{3} \sin \theta & \cos \theta+v_{3} \sin \theta & -\left(v_{2}-v_{1}\right) \sin \theta \\
-v_{3} \sin \theta & v_{2} \sin \theta & -v_{1} \sin \theta & \cos \theta
\end{array}\right] } \\
= & {\left[\begin{array}{cccc}
\cos (-\theta) & \left(v_{2}-v_{1}\right) \sin (-\theta) & v_{1} \sin (-\theta) & v_{3} \sin (-\theta) \\
v_{1} \sin (-\theta) & \cos (-\theta)+v_{3} \sin (-\theta) & 0 & -v_{1} \sin (-\theta) \\
v_{2} \sin (-\theta) & v_{3} \sin (-\theta) & \cos (-\theta)-v_{3} \sin (-\theta) & \left(v_{2}-v_{1}\right) \sin (-\theta) \\
v_{3} \sin (-\theta) & -v_{2}-\sin (-\theta) & v_{1} \sin (-\theta) & \cos (-\theta)
\end{array}\right] . }
\end{aligned}
$$

Then by using mathematical induction method, we obtain

$$
\mathbf{Z}_{R}^{-n}=\left[\begin{array}{cccc}
\cos (-n \theta) & \left(v_{2}-v_{1}\right) \sin (-n \theta) & v_{1} \sin (-n \theta) & v_{3} \sin (-n \theta) \\
v_{1} \sin (-n \theta) & \cos (-n \theta)+v_{3} \sin (-n \theta) & 0 & -v_{1} \sin (-n \theta) \\
v_{2} \sin (-n \theta) & v_{3} \sin (-n \theta) & \cos (-n \theta)-v_{3} \sin (-n \theta) & \left(v_{2}-v_{1}\right) \sin (-n \theta) \\
v_{3} \sin (-n \theta) & -v_{2} \sin (-n \theta) & v_{1} \sin (-n \theta) & \cos (-n \theta)
\end{array}\right] .
$$

Finally, the proof is completed. 
Theorem 2. Let

$$
\boldsymbol{Z}_{R}=\left[\begin{array}{cccc}
\sinh \theta & \left(v_{2}-v_{1}\right) \cosh \theta & v_{1} \cosh \theta & v_{3} \cosh \theta \\
v_{1} \cosh \theta & \sinh \theta+v_{3} \cosh \theta & 0 & -v_{1} \cosh \theta \\
v_{2} \cosh \theta & v_{3} \cosh \theta & \sinh \theta-v_{3} \cosh \theta & \left(v_{2}-v_{1}\right) \cosh \theta \\
v_{3} \cosh \theta & -v_{2} \cosh \theta & v_{1} \cosh \theta & \sinh \theta
\end{array}\right]
$$

be a right matrix representation for a spacelike hyperbolic hybrid number. Then, the nth power of the matrix is given as follows:

If $n$ is an odd number, then

$$
\mathbf{Z}_{R}^{n}=\left[\begin{array}{cccc}
\sinh n \theta & \left(v_{2}-v_{1}\right) \cosh n \theta & v_{1} \cosh n \theta & v_{3} \cosh n \theta \\
v_{1} \cosh n \theta & \sinh n \theta+v_{3} \cosh n \theta & 0 & -v_{1} \cosh n \theta \\
v_{2} \cosh n \theta & v_{3} \cosh n \theta & \sinh n \theta-v_{3} \cosh n \theta & \left(v_{2}-v_{1}\right) \cosh n \theta \\
v_{3} \cosh n \theta & -v_{2} \cosh n \theta & v_{1} \cosh n \theta & \sinh n \theta
\end{array}\right] .
$$

If $n$ is an even number, then

$$
\mathbf{Z}_{R}^{n}=\left[\begin{array}{cccc}
\cosh n \theta & \left(v_{2}-v_{1}\right) \sinh n \theta & v_{1} \sinh n \theta & v_{3} \sin n \theta \\
v_{1} \sinh n \theta & \cosh n \theta+v_{3} \sinh n \theta & 0 & -v_{1} \sinh n \theta \\
v_{2} \sinh n \theta & v_{3} \sinh n \theta & \cosh n \theta-v_{3} \sinh n \theta & \left(v_{2}-v_{1}\right) \sinh n \theta \\
v_{3} \sinh n \theta & -v_{2} \sinh n \theta & v_{1} \sinh n \theta & \cosh n \theta
\end{array}\right] .
$$

Proof. The proof can be calculated by following the same steps at the proof of the Theorem 1 and by using the Lemma 2.

Theorem 3. Let

$$
\mathbf{Z}_{R}= \pm\left[\begin{array}{cccc}
\cosh \theta & \left(v_{2}-v_{1}\right) \sinh \theta & v_{1} \sinh \theta & v_{3} \sinh \theta \\
v_{1} \sinh \theta & \cosh \theta+v_{3} \sinh \theta & 0 & -v_{1} \sinh \theta \\
v_{2} \sinh \theta & v_{3} \sinh \theta & \cosh \theta-v_{3} \sin \theta & \left(v_{2}-v_{1}\right) \sinh \theta \\
v_{3} \sinh \theta & -v_{2} \sinh \theta & v_{1} \sinh \theta & \cosh \theta
\end{array}\right]
$$

be a right matrix representation for a unit timelike hyperbolic hybrid number. Then the nth power of the matrix is given as follows:

$$
\mathbf{Z}_{R}^{n}=( \pm 1)^{n}\left[\begin{array}{cccc}
\cosh n \theta & \left(v_{2}-v_{1}\right) \sinh n \theta & v_{1} \sinh n \theta & v_{3} \sin n \theta \\
v_{1} \sinh n \theta & \cosh n \theta+v_{3} \sinh n \theta & 0 & -v_{1} \sinh n \theta \\
v_{2} \sinh n \theta & v_{3} \sinh n \theta & \cosh n \theta-v_{3} \sinh n \theta & \left(v_{2}-v_{1}\right) \sinh n \theta \\
v_{3} \sinh n \theta & -v_{2} \sinh n \theta & v_{1} \sinh n \theta & \cosh n \theta
\end{array}\right] .
$$

Proof. The proof can be seen clearly by using the same way of Theorem 1 and Lemma 3.

Theorem 4. Let

$$
\mathbf{Z}_{R}=\left[\begin{array}{cccc}
\epsilon & \left(v_{2}-v_{1}\right) & v_{1} & v_{3} \\
v_{1} & \epsilon+v_{3} & 0 & -v_{1} \\
v_{2} & v_{3} & \epsilon-v_{3} & \left(v_{2}-v_{1}\right) \\
v_{3} & -v_{2} & v_{1} & \epsilon
\end{array}\right]
$$

be a right matrix representation for a parabolic hybrid number. Then the nth power of the matrix is given as follows:

$$
\mathbf{Z}_{R}^{n}=\left[\begin{array}{cccc}
\epsilon^{n} & n \epsilon^{n-1}\left(v_{2}-v_{1}\right) & n \epsilon^{n-1} v_{1} & n \epsilon^{n-1} v_{3} \\
n \epsilon^{n-1} v_{1} & \epsilon^{n}+n \epsilon^{n-1} v_{3} & 0 & -n \epsilon^{n-1} v_{1} \\
n \epsilon^{n-1} v_{2} & n \epsilon^{n-1} v_{3} & \epsilon^{n}-n \epsilon^{n-1} v_{3} & n \epsilon^{n-1}\left(v_{2}-v_{1}\right) \\
n \epsilon^{n-1} v_{3} & n \epsilon^{n-1} v_{2} & n \epsilon^{n-1} v_{1} & \epsilon^{n}
\end{array}\right] .
$$


Proof. We can write that $\mathbf{Z}_{R}=\epsilon \mathbf{I}_{4}+\mathbf{A}$ where $\mathbf{I}_{4}$ is a 4 by 4 unit matrix and

$$
\mathbf{A}=\left[\begin{array}{cccc}
0 & v_{2}-v_{1} & v_{1} & v_{3} \\
v_{1} & v_{3} & 0 & -v_{1} \\
v_{2} & v_{3} & -v_{3} & v_{2}-v_{1} \\
v_{3} & -v_{2} & v_{1} & 0
\end{array}\right]
$$

Since $\mathbf{A}^{2}=0$, then we get

$$
\mathbf{Z}_{R}^{n}=\epsilon^{n} \mathbf{I}_{4}+n \epsilon^{n-1} \mathbf{A}
$$

with the induction method over $n$.

Moreover, the theorem given above can be written in the following form with cosine and sine Galilean trigonometric functions.

Theorem 5. Let

$$
\boldsymbol{Z}_{R}=\left[\begin{array}{cccc}
\cos _{g} \theta & \left(v_{2}-v_{1}\right) \sin _{g} \theta & v_{1} \sin _{g} \theta & v_{3} \sin _{g} \theta \\
v_{1} \sin _{g} \theta & \cos \theta+v_{3} \sin _{g} \theta & 0 & -v_{1} \sin _{g} \theta \\
v_{2} \sin _{g} \theta & v_{3} \sin _{g} \theta & \cos _{g} \theta-v_{3} \sin _{g} \theta & \left(v_{2}-v_{1}\right) \sin _{g} \theta \\
v_{3} \sin _{g} \theta & -v_{2} \sin _{g} \theta & v_{1} \sin _{g} \theta & \cos g
\end{array}\right]
$$

be a right matrix representation for a unit parabolic hybrid number and $c \neq 0$. Then, the nth power of the matrix is given as follows:

$$
Z_{R}^{n}=\left[\begin{array}{cccc}
\cos _{g} n \theta & \left(v_{2}-v_{1}\right) \sin _{g} n \theta & v_{1} \sin g n & v_{3} \sin g n \theta \\
v_{1} \sin _{g} n \theta & \cos g n \theta+v_{3} \sin _{g} n \theta & 0 & -v_{1} \sin g n \\
v_{2} \sin _{g} n \theta & v_{3} \sin _{g} n \theta & \cos g \theta-v_{3} \sin g \theta & \left(v_{2}-v_{1}\right) \sin g n \theta \\
v_{3} \sin _{g} n \theta & -v_{2} \sin _{g} n \theta & v_{1} \sin _{g} n \theta & \cos _{g} n \theta
\end{array}\right] .
$$

Proof. The proof is easy through the Theorem 1 and Lemma 4.

Theorem 6. Let

$$
\mathbf{Z}_{R}=\left[\begin{array}{cccc}
a & a\left(v_{2}-v_{1}\right) & a v_{1} & a v_{3} \\
a v_{1} & a+a v_{3} & 0 & -a v_{1} \\
a v_{2} & a v_{3} & a-a v_{3} & a\left(v_{2}-v_{1}\right) \\
a v_{3} & -a v_{2} & a v_{1} & a
\end{array}\right]
$$

be a right matrix representation for a lightlike hybrid number. Then the nth power of the matrix is given as follows:

$$
Z_{R}^{n}=\left[\begin{array}{cccc}
a^{n} 2^{n-1} & a^{n} 2^{n-1}\left(v_{2}-v_{1}\right) & a^{n} 2^{n-1} v_{1} & a^{n} 2^{n-1} v_{3} \\
a^{n} 2^{n-1} v_{1} & a^{n} 2^{n-1}+a^{n} 2^{n-1} v_{3} & 0 & -a^{n} 2^{n-1} v_{1} \\
a^{n} 2^{n-1} v_{2} & a^{n} 2^{n-1} v_{3} & a^{n} 2^{n-1}-a^{n} 2^{n-1} v_{3} & a^{n} 2^{n-1}\left(v_{2}-v_{1}\right) \\
a^{n} 2^{n-1} v_{3} & -a^{n} 2^{n-1} v_{2} & a^{n} 2^{n-1} v_{1} & a^{n} 2^{n-1}
\end{array}\right] .
$$

Proof. The proof is easy through the Theorem 1.

\section{Euler Formula for the Matrices of Hybrid Numbers}

This section is devoted to compute Euler formulas for matrices of hybrid numbers. For this purpose, let us consider a matrix as below:

$$
\mathbf{A}=\left[\begin{array}{cccc}
0 & v_{2}-v_{1} & v_{1} & v_{3} \\
v_{1} & v_{3} & 0 & -v_{1} \\
v_{2} & v_{3} & -v_{3} & v_{2}-v_{1} \\
v_{3} & -v_{2} & v_{1} & 0
\end{array}\right]
$$


Then

$$
\mathbf{A}^{2}=\left(v_{3}^{2}+2 v_{1} v_{2}-v_{1}^{2}\right)\left[\begin{array}{llll}
1 & 0 & 0 & 0 \\
0 & 1 & 0 & 0 \\
0 & 0 & 1 & 0 \\
0 & 0 & 0 & 1
\end{array}\right] .
$$

Theorem 7. Let $Z$ be a pure hybrid elliptic number. Then, $e^{\theta \mathbf{Z}_{R}}=\cos \theta \mathbf{I}_{4}+\sin \theta \mathbf{Z}_{R}$.

Proof. The right matrix representation of the pure unit hybrid number is

$$
\mathbf{Z}_{R}=\left[\begin{array}{cccc}
0 & v_{2}-v_{1} & v_{1} & v_{3} \\
v_{1} & v_{3} & 0 & -v_{1} \\
v_{2} & v_{3} & -v_{3} & v_{2}-v_{1} \\
v_{3} & -v_{2} & v_{1} & 0
\end{array}\right]
$$

Since

$$
e^{\theta \mathbf{Z}_{R}}=I_{4}+\theta \mathbf{Z}_{R}+\frac{(\theta)^{2}}{2} \mathbf{Z}_{R}^{2}+\frac{(\theta)^{3}}{3 !} \mathbf{Z}_{R}^{3}+\frac{(\theta)^{4}}{4 !} \mathbf{Z}_{R}^{4}+\ldots
$$

and $\mathbf{Z}_{R}^{2}=-I_{4}$, then we write $e^{\theta \mathbf{Z}_{R}}=\cos \theta \mathbf{I}_{4}+\sin \theta \mathbf{Z}_{R}$.

Theorem 8. Let $\mathrm{Z}$ be a pure hyperbolic hybrid number. Then,

$$
e^{\theta \mathbf{Z}_{R}}=\cosh \theta \mathbf{I}_{4}+\sinh \theta \mathbf{Z}_{R} .
$$

Proof. The right matrix representation of the pure unit hyperbolic hybrid number is

$$
\mathbf{Z}_{R}=\left[\begin{array}{cccc}
0 & v_{2}-v_{1} & v_{1} & v_{3} \\
v_{1} & v_{3} & 0 & -v_{1} \\
v_{2} & v_{3} & -v_{3} & v_{2}-v_{1} \\
v_{3} & -v_{2} & v_{1} & 0
\end{array}\right] .
$$

Since

$$
e^{\theta \mathbf{Z}_{R}}=I_{4}+\theta \mathbf{Z}_{R}+\frac{(\theta)^{2}}{2} \mathbf{Z}_{R}^{2}+\frac{(\theta)^{3}}{3 !} \mathbf{Z}_{R}^{3}+\frac{(\theta)^{4}}{4 !} \mathbf{Z}_{R}^{4}+\ldots
$$

and $\mathbf{Z}_{R}^{2}=I_{4}$, we get $e^{\theta \mathbf{Z}_{R}}=\cosh \theta \mathbf{I}_{4}+\sinh \theta \mathbf{Z}_{R}$.

Theorem 9. Let $Z$ be a pure hybrid parabolic number. Then,

$$
e^{\theta \mathbf{Z}_{R}}=\mathbf{I}_{4}+\theta \mathbf{Z}_{R} .
$$

Proof. The right matrix representation of the pure parabolic hybrid number is

$$
\mathbf{Z}_{R}=\left[\begin{array}{cccc}
0 & v_{2}-v_{1} & v_{1} & v_{3} \\
v_{1} & v_{3} & 0 & -v_{1} \\
v_{2} & v_{3} & -v_{3} & v_{2}-v_{1} \\
v_{3} & -v_{2} & v_{1} & 0
\end{array}\right] .
$$

Since

$$
e^{\theta \mathbf{Z}_{R}}=I_{4}+\theta \mathbf{Z}_{R}+\frac{(\theta)^{2}}{2} \mathbf{Z}_{R}^{2}+\frac{(\theta)^{3}}{3 !} \mathbf{Z}_{R}^{3}+\frac{(\theta)^{4}}{4 !} \mathbf{Z}_{R}^{4}+\ldots
$$

and $\mathbf{Z}_{R}^{2}=0_{4}$, we find $e^{\theta \mathbf{Z}_{R}}=\mathbf{I}_{4}+\theta \mathbf{Z}_{R}$. 


\section{Roots of the Matrices of Hybrid Numbers}

In this section, we give the roots of the matrices of hybrid numbers. The right matrix representation in the general form for a unit elliptic hybrid number are given the following matrix:

$$
\mathbf{Z}_{R}=\left[\begin{array}{cccc}
\cos \beta & \left(v_{2}-v_{1}\right) \sin \beta & v_{1} \sin \beta & v_{3} \sin \beta \\
v_{1} \sin \beta & \cos \beta+v_{3} \sin \beta & 0 & -v_{1} \sin \beta \\
v_{2} \sin \beta & v_{3} \sin \beta & \cos \beta-v_{3} \beta & \left(v_{2}-v_{1}\right) \sin \beta \\
v_{3} \sin \beta & -v_{2} \sin \beta & v_{1} \sin \beta & \cos \beta
\end{array}\right]
$$

where $\beta=\theta+2 l \pi$ for $l \in \mathbb{Z}$. Thus, there exist $n$th roots;

$$
\mathbf{X}_{s}=\left[\begin{array}{cccc}
\cos \beta_{s} & \left(v_{2}-v_{1}\right) \sin \beta_{s} & v_{1} \sin \beta_{s} & v_{3} \sin \beta_{s} \\
v_{1} \sin \beta_{s} & \cos \beta_{s}+v_{3} \sin \beta_{s} & 0 & -v_{1} \sin \beta_{s} \\
v_{2} \sin \beta_{s} & v_{3} \sin \beta_{s} & \cos \beta_{s}-v_{3} \beta_{s} & \left(v_{2}-v_{1}\right) \sin \beta_{s} \\
v_{3} \sin \beta_{s} & -v_{2} \sin \beta_{s} & v_{1} \sin \beta_{s} & \cos \beta_{s}
\end{array}\right],
$$

where $\beta_{s}=\frac{\theta+2 s \pi}{n}$ and $s=0,1, \ldots, n-1$, of the equation $\mathbf{X}^{n}=\mathbf{Z}_{R}$. Especially, if we take $n=2$, then we get

$$
\mathbf{X}_{0}=\left[\begin{array}{cccc}
\cos \frac{\theta}{2} & \left(v_{2}-v_{1}\right) \sin \frac{\theta}{2} & v_{1} \sin \frac{\theta}{2} & v_{3} \sin \frac{\theta}{2} \\
v_{1} \sin \frac{\theta}{2} & \cos \frac{\theta}{2}+v_{3} \sin \frac{\theta}{2} & 0 & -v_{1} \sin \frac{\theta}{2} \\
v_{2} \sin \frac{\theta}{2} & v_{3} \sin \frac{\theta}{2} & \cos \frac{\theta}{2}-v_{3} \frac{\theta}{2} & \left(v_{2}-v_{1}\right) \sin \frac{\theta}{2} \\
v_{3} \sin \frac{\theta}{2} & -v_{2} \sin \frac{\theta}{2} & v_{1} \sin \frac{\theta}{2} & \cos \frac{\theta}{2}
\end{array}\right]
$$

and

$$
\mathbf{X}_{1}=\left[\begin{array}{cccc}
-\cos \frac{\theta}{2} & \left(v_{1}-v_{2}\right) \sin \frac{\theta}{2} & -v_{1} \sin \frac{\theta}{2} & -v_{3} \sin \frac{\theta}{2} \\
-v_{1} \sin \frac{\theta}{2} & -\cos \frac{\theta}{2}-v_{3} \sin \frac{\theta}{2} & 0 & v_{1} \sin \frac{\theta}{2} \\
-v_{2} \sin \frac{\theta}{2} & -v_{3} \sin \frac{\theta}{2} & -\cos \frac{\theta}{2}+v_{3} \frac{\theta}{2} & \left(v_{1}-v_{2}\right) \sin \frac{\theta}{2} \\
-v_{3} \sin \frac{\theta}{2} & v_{2} \sin \frac{\theta}{2} & -v_{1} \sin \frac{\theta}{2} & -\cos \frac{\theta}{2}
\end{array}\right]
$$

of the equation $\mathbf{X}^{2}=\mathbf{Z}_{R}$. Hence $\mathbf{X}_{0}+\mathbf{X}_{1}=0$.

Similarly, we have the following roots of the equation $\mathbf{X}^{n}=\mathbf{Z}_{R}$, where $\mathbf{Z}_{R}$ is the right matrix representation form of the non lightlike hybrid number.

(A) If $n$ is an even number for a unit hybrid number $Z=\cosh \theta+V \sinh \theta$, then there exist 4 distinct roots:

$$
\begin{aligned}
& \mathbf{X}_{0}=\left[\begin{array}{cccc}
\sinh \beta & \left(v_{2}-v_{1}\right) \cosh \beta & v_{1} \cosh \beta & v_{3} \cosh \beta \\
v_{1} \cosh \beta & \sinh \beta+v_{3} \cosh \beta & 0 & -v_{1} \cosh \beta \\
v_{2} \cosh \beta & v_{3} \cosh \beta & \sinh \beta-v_{3} \cosh \beta & \left(v_{2}-v_{1}\right) \cosh \beta \\
v_{3} \cosh \beta & -v_{2} \cosh \beta & v_{1} \cosh \beta & \sinh \beta
\end{array}\right], \\
& \mathbf{X}_{1}=-\left[\begin{array}{cccc}
\sinh \beta & \left(v_{2}-v_{1}\right) \cosh \beta & v_{1} \cosh \beta & v_{3} \cosh \beta \\
v_{1} \cosh \beta & \sinh \beta+v_{3} \cosh \beta & 0 & -v_{1} \cosh \beta \\
v_{2} \cosh \beta & v_{3} \cosh \beta & \sinh \beta-v_{3} \cosh \beta & \left(v_{2}-v_{1}\right) \cosh \beta \\
v_{3} \cosh \beta & -v_{2} \cosh \beta & v_{1} \cosh \beta & \sinh \beta
\end{array}\right], \\
& \mathbf{X}_{2}=\left[\begin{array}{cccc}
\cosh \beta & \left(v_{2}-v_{1}\right) \sinh \beta & v_{1} \sinh \beta & v_{3} \sinh \beta \\
v_{1} \sinh \beta & \cosh \beta+v_{3} \sinh \beta & 0 & -v_{1} \sinh \beta \\
v_{2} \sinh \beta & v_{3} \sinh \beta & \cosh \beta-v_{3} \sinh \beta & \left(v_{2}-v_{1}\right) \sinh \beta \\
v_{3} \sinh \beta & -v_{2} \sinh \beta & v_{1} \sinh \beta & \cosh \beta
\end{array}\right], \\
& \mathbf{X}_{3}=-\left[\begin{array}{cccc}
\cosh \beta & \left(v_{2}-v_{1}\right) \sinh \beta & v_{1} \sinh \beta & v_{3} \sinh \beta \\
v_{1} \sinh \beta & \cosh \beta+v_{3} \sinh \beta & 0 & -v_{1} \sinh \beta \\
v_{2} \sinh \beta & v_{3} \sinh \beta & \cosh \beta-v_{3} \sinh \beta & \left(v_{2}-v_{1}\right) \sinh \beta \\
v_{3} \sinh \beta & -v_{2} \sinh \beta & v_{1} \sinh \beta & \cosh \beta
\end{array}\right] \text {, }
\end{aligned}
$$


where $\beta=\frac{\theta}{n}$.

If $n$ is an odd number for a unit hybrid number $Z=\cosh \theta+V \sinh \theta$, then there exist only one root:

$$
\mathbf{X}_{0}=\left[\begin{array}{cccc}
\cosh \beta & \left(v_{2}-v_{1}\right) \sinh \beta & v_{1} \sinh \beta & v_{3} \sinh \beta \\
v_{1} \sinh \beta & \cosh \beta+v_{3} \sinh \beta & 0 & -v_{1} \sinh \beta \\
v_{2} \sinh \beta & v_{3} \sinh \beta & \cosh \beta-v_{3} \sinh \beta & \left(v_{2}-v_{1}\right) \sinh \beta \\
v_{3} \sinh \beta & -v_{2} \sinh \beta & v_{1} \sinh \beta & \cosh \beta
\end{array}\right]
$$

where $\beta=\frac{\theta}{n}$.

(B) If $n$ is an even number for a unit hybrid number $Z=\sinh \theta+V \cosh \theta$, there no roots.

If $n$ is odd number for a unit hybrid number $Z=\sinh \theta+V \cosh \theta$,

$$
\mathbf{X}_{0}=\left[\begin{array}{cccc}
\sinh \beta & \left(v_{2}-v_{1}\right) \cosh \beta & v_{1} \cosh \beta & v_{3} \cosh \beta \\
v_{1} \cosh \beta & \sinh \beta+v_{3} \cosh \beta & 0 & -v_{1} \cosh \beta \\
v_{2} \cosh \beta & v_{3} \cosh \beta & \sinh \beta-v_{3} \cosh \beta & \left(v_{2}-v_{1}\right) \cosh \beta \\
v_{3} \cosh \beta & -v_{2} \cosh \beta & v_{1} \cosh \beta & \sinh \beta
\end{array}\right] \text {, }
$$

where $\beta=\frac{\theta}{n}$.

(C) If $n$ is an even number for a unit hybrid number $Z=-(\cosh \theta+V \sinh \theta)$, there is no roots.

If $n$ is an odd number for a unit hybrid number $Z=-(\cosh \theta+V \sinh \theta)$, there is only one root

$$
\mathbf{X}_{0}=-\left[\begin{array}{cccc}
\cosh \beta & \left(v_{2}-v_{1}\right) \sinh \beta & v_{1} \sinh \beta & v_{3} \sinh \beta \\
v_{1} \sinh \beta & \cosh \beta+v_{3} \sinh \beta & 0 & -v_{1} \sinh \beta \\
v_{2} \sinh \beta & v_{3} \sinh \beta & \cosh \beta-v_{3} \sinh \beta & \left(v_{2}-v_{1}\right) \sinh \beta \\
v_{3} \sinh \beta & -v_{2} \sinh \beta & v_{1} \sinh \beta & \cosh \beta
\end{array}\right],
$$

where $\beta=\frac{\theta}{n}$.

(D) If $n$ is an even number for a unit hybrid number $Z=-(\sinh \theta+V \cosh \theta)$, there no roots.

If $n$ is an odd number for a unit hybrid number $Z=-(\sinh \theta+V \cosh \theta)$,

$$
\mathbf{X}_{0}=-\left[\begin{array}{cccc}
\sinh \beta & \left(v_{2}-v_{1}\right) \cosh \beta & v_{1} \cosh \beta & v_{3} \cosh \beta \\
v_{1} \cosh \beta & \sinh \beta+v_{3} \cosh \beta & 0 & -v_{1} \cosh \beta \\
v_{2} \cosh \beta & v_{3} \cosh \beta & \sinh \beta-v_{3} \cosh \beta & \left(v_{2}-v_{1}\right) \cosh \beta \\
v_{3} \cosh \beta & -v_{2} \cosh \beta & v_{1} \cosh \beta & \sinh \beta
\end{array}\right],
$$

where $\beta=\frac{\theta}{n}$.

Moreover, we have the following roots of the equation $\mathbf{X}^{n}=\mathbf{Z}_{R}$, where $\mathbf{Z}_{R}$ is the right matrix representation form of the unit parabolic hybrid number. If $n$ is an odd number, then we have one root as follows:

$$
\mathbf{X}_{0}=\left[\begin{array}{cccc}
\epsilon & \frac{1}{n}\left(v_{2}-v_{1}\right) & \frac{1}{n} v_{1} & \frac{1}{n} v_{3} \\
\frac{1}{n} v_{1} & \epsilon+\frac{1}{n} v_{3} & 0 & -\frac{1}{n} v_{1} \\
\frac{1}{n} v_{2} & \frac{1}{n} v_{3} & \epsilon-\frac{1}{n} v_{3} & \frac{1}{n}\left(v_{2}-v_{1}\right) \\
\frac{1}{n} v_{3} & -\frac{1}{n} v_{2} & \frac{1}{n} v_{1} & \epsilon
\end{array}\right] .
$$

If $n$ is an even number, then we have two roots as follows:

$$
\mathbf{X}_{0}=\left[\begin{array}{cccc}
\epsilon & \frac{1}{n}\left(v_{2}-v_{1}\right) & \frac{1}{n} v_{1} & \frac{1}{n} v_{3} \\
\frac{1}{n} v_{1} & \epsilon+\frac{1}{n} v_{3} & 0 & -\frac{1}{n} v_{1} \\
\frac{1}{n} v_{2} & \frac{1}{n} v_{3} & \epsilon-\frac{1}{n} v_{3} & \frac{1}{n}\left(v_{2}-v_{1}\right) \\
\frac{1}{n} v_{3} & -\frac{1}{n} v_{2} & \frac{1}{n} v_{1} & \epsilon
\end{array}\right]
$$


and

$$
\mathbf{X}_{1}=-\left[\begin{array}{cccc}
\epsilon & \frac{1}{n}\left(v_{2}-v_{1}\right) & \frac{1}{n} v_{1} & \frac{1}{n} v_{3} \\
\frac{1}{n} v_{1} & \epsilon+\frac{1}{n} v_{3} & 0 & -\frac{1}{n} v_{1} \\
\frac{1}{n} v_{2} & \frac{1}{n} v_{3} & \epsilon-\frac{1}{n} v_{3} & \frac{1}{n}\left(v_{2}-v_{1}\right) \\
\frac{1}{n} v_{3} & -\frac{1}{n} v_{2} & \frac{1}{n} v_{1} & \epsilon
\end{array}\right],
$$

where $\epsilon>0$. Otherwise there exists no roots. Especially, let $\mathbf{Z}_{R}$ be the right matrix representation form of the unit parabolic hybrid number and $c \neq 0$. If $n$ is an odd number, then we have one root as follow:

$$
\mathbf{X}_{0}=\left[\begin{array}{cccc}
\cos _{g} \beta & \left(v_{2}-v_{1}\right) \sin _{g} \beta & v_{1} \sin _{g} \beta & v_{3} \sin _{g} \beta \\
v_{1} \sin _{g} \beta & \cos _{g} \beta+v_{3} \sin _{g} \beta & 0 & -v_{1} \sin _{g} \beta \\
v_{2} \sin _{g} \beta & v_{3} \sin _{g} \beta & \cos _{g} \beta-v_{3} \sin _{g} \beta & \left(v_{2}-v_{1}\right) \sin _{g} \beta \\
v_{3} \sin _{g} \beta & -v_{2} \sin _{g} \beta & v_{1} \sin _{g} \beta & \cos \beta
\end{array}\right],
$$

where $\beta=\frac{\theta}{n}$. If $n$ is an even number, then we have two roots as follow:

$$
\mathbf{X}_{0}=\left[\begin{array}{cccc}
\cos _{g} \beta & \left(v_{2}-v_{1}\right) \sin _{g} \beta & v_{1} \sin _{g} \beta & v_{3} \sin _{g} \beta \\
v_{1} \sin _{g} \beta & \cos _{g} \beta+v_{3} \sin _{g} \beta & 0 & -v_{1} \sin _{g} \beta \\
v_{2} \sin _{g} \beta & v_{3} \sin _{g} \beta & \cos _{g} \beta-v_{3} \sin n_{g} \beta & \left(v_{2}-v_{1}\right) \sin _{g} \beta \\
v_{3} \sin _{g} \beta & -v_{2} \sin _{g} \beta & v_{1} \sin _{g} \beta & \cos _{g} \beta
\end{array}\right]
$$

and

$$
\mathbf{X}_{1}=-\left[\begin{array}{cccc}
\cos _{g} \beta & \left(v_{2}-v_{1}\right) \sin _{g} \beta & v_{1} \sin _{g} \beta & v_{3} \sin _{g} \beta \\
v_{1} \sin _{g} \beta & \cos _{g} \beta+v_{3} \sin _{g} \beta & 0 & -v_{1} \sin _{g} \beta \\
v_{2} \sin _{g} \beta & v_{3} \sin _{g} \beta & \cos _{g} \beta-v_{3} \sin _{g} \beta & \left(v_{2}-v_{1}\right) \sin _{g} \beta \\
v_{3} \sin _{g} \beta & -v_{2} \sin _{g} \beta & v_{1} \sin _{g} \beta & \cos _{g} \beta
\end{array}\right],
$$

where $\beta=\frac{\theta}{n}$ and $\cos _{g} \theta>0$. Otherwise, there exists no roots.

Finally, we have the following roots of the equation $\mathbf{X}^{n}=\mathbf{Z}_{R}$, where $\mathbf{Z}_{R}$ is the right matrix representation form of the lightlike hybrid number. If $n$ is an odd number, then we have only one root

$$
\mathbf{X}_{0}=\left[\begin{array}{cccc}
\mu & \mu\left(v_{2}-v_{1}\right) & \mu v_{1} & \mu v_{3} \\
\mu v_{1} & \mu+\mu v_{3} & 0 & -\mu v_{1} \\
\mu v_{2} & \mu v_{3} & \mu-\mu v_{3} & \mu\left(v_{2}-v_{1}\right) \\
\mu v_{3} & -\mu v_{2} & \mu v_{1} & \mu
\end{array}\right]
$$

where $\mu=\frac{\sqrt[n]{2 a}}{2}$.

If $n$ is an even number, then we have two roots

$$
\mathbf{X}_{0}=\left[\begin{array}{cccc}
\mu & \mu\left(v_{2}-v_{1}\right) & \mu v_{1} & \mu v_{3} \\
\mu v_{1} & \mu+\mu v_{3} & 0 & -\mu v_{1} \\
\mu v_{2} & \mu v_{3} & \mu-\mu v_{3} & \mu\left(v_{2}-v_{1}\right) \\
\mu v_{3} & -\mu v_{2} & \mu v_{1} & \mu
\end{array}\right]
$$

where $\mu=\frac{\sqrt[n]{2 a}}{2}$ and

$$
\mathbf{X}_{1}=-\left[\begin{array}{cccc}
\mu & \mu\left(v_{2}-v_{1}\right) & \mu v_{1} & \mu v_{3} \\
\mu v_{1} & \mu+\mu v_{3} & 0 & -\mu v_{1} \\
\mu v_{2} & \mu v_{3} & \mu-\mu v_{3} & \mu\left(v_{2}-v_{1}\right) \\
\mu v_{3} & -\mu v_{2} & \mu v_{1} & \mu
\end{array}\right]
$$

where $\mu=\frac{\sqrt[n]{2 a}}{2}$. 


\section{Applications}

In this section, we give some illustrative examples.

Example 1. Let us consider any hybrid number $Z=6 i+\varepsilon+\sqrt{23}$. By obtaining $C(z)=1$ and $C_{\varepsilon}(z)=-1$, the characterization of $Z$ is denoted by a unit timelike elliptic hybrid number. Therefore, the polar form of $\mathrm{Z}$ is:

$$
Z=V,
$$

where $V=6 i+\varepsilon+\sqrt{23}$. The matrix representation of $Z$ is

$$
Z_{R}=\left(\begin{array}{cccc}
0 & -5 & 6 & \sqrt{23} \\
6 & \sqrt{23} & 0 & -6 \\
1 & \sqrt{23} & -\sqrt{23} & -5 \\
\sqrt{23} & -1 & 6 & 0
\end{array}\right)
$$

Computing $Z_{R}^{-3}$, we obtain

$$
Z_{R}^{-3}=\left(\begin{array}{cccc}
0 & -5 & 6 & \sqrt{23} \\
6 & \sqrt{23} & 0 & -6 \\
1 & \sqrt{23} & -\sqrt{23} & -5 \\
\sqrt{23} & -1 & 6 & 0
\end{array}\right)
$$

By exploiting De Moivre's formula for negative powers, we obtain the following matrix for $n=-3:$

$$
Z_{R}^{-3}=\left(\begin{array}{cccc}
\cos (-3 \theta) & \left(v_{2}-v_{1}\right) \sin (-3 \theta) & v_{1} \sin (-3 \theta) & v_{3} \sin (-3 \theta) \\
v_{1} \sin (-3 \theta) & \cos (-3 \theta)+v_{3} \sin (-3 \theta) & 0 & -v_{1} \sin (-3 \theta) \\
v_{2} \sin (-3 \theta) & v_{3} \sin (-3 \theta) & \cos (-3 \theta)-v_{3} \sin (-3 \theta) & \left(v_{2}-v_{1}\right) \sin (-3 \theta) \\
v_{3} \sin (-3 \theta) & -v_{2} \sin (-3 \theta) & v_{1} \sin (-3 \theta) & \cos (-3 \theta)
\end{array}\right),
$$

where $v_{1}=6, v_{2}=1, v_{3}=\sqrt{23}$ and $\theta=\frac{\pi}{2}$. Hence, its numerical value is found the same matrix as above.

Example 2. Let us consider the hybrid number as $Z=\sqrt{5}+2 h$. Since $C(Z)=1$ and $C_{\varepsilon}(Z)=4$, the hybrid number is called unit timelike hyperbolic hybrid number. The polar form of $Z$ is

$$
Z= \pm(\sqrt{5}+V \cdot 2),
$$

where $V=h$.

$$
Z_{R}= \pm\left(\begin{array}{cccc}
2.236067977 & 0 & 0 & 2 \\
0 & 4.236067977 & 0 & 0 \\
0 & 2 & 0.236067977 & 0 \\
2 & 0 & 0 & 2.236067977
\end{array}\right)
$$

where $v_{1}=v_{2}=0$ and $v_{3}=1, \theta=\operatorname{arc}(\cosh (\sqrt{5}))$. For $n=3$ :

$$
Z_{R}^{3}=( \pm 1)^{3}\left(\begin{array}{cccc}
38.0131 & 0 & 0 & 37.9999 \\
0 & 76.0131 & 0 & 0 \\
0 & 37.9999 & 0.01311 & 0 \\
37.9999 & 0 & 0 & 38.0131
\end{array}\right)
$$

Example 3. Let us consider any hybrid number as $Z=1+i+\varepsilon+h$. Since $C(Z)=-1$ and $C_{\varepsilon}(Z)=2, Z$ is a unit spacelike hyperbolic hybrid number. The polar form of $Z$ is denoted by

$$
Z=1+V \sqrt{2},
$$


where $V=\frac{i+\varepsilon+h}{\sqrt{2}}$. The matrix representation of $Z$ is

$$
Z_{R}=\left(\begin{array}{cccc}
1 & 0 & 1 & 1 \\
1 & 2 & 0 & -1 \\
1 & 1 & 0 & 0 \\
1 & -1 & 1 & 1
\end{array}\right)
$$

Let us compute the power of the matrix $Z_{R}$ for $n=7$ :

$$
\left(Z_{R}\right)^{7}=\left(\begin{array}{cccc}
239 & 0 & 169 & 169 \\
169 & 408 & 0 & -169 \\
169 & 169 & 70 & 0 \\
169 & -169 & 169 & 239
\end{array}\right) .
$$

This can be stated with De Moivre's formula as below:

$$
\left(Z_{R}\right)^{7}=\left(\begin{array}{cccc}
\sinh (7 \theta) & \left(v_{2}-v_{1}\right) \cosh (7 \theta) & v_{1} \cosh (7 \theta) & v_{3} \cosh (7 \theta) \\
v_{1} \cosh (7 \theta) & \sinh (7 \theta)+v_{3} \cosh (7 \theta) & 0 & -v_{1} \cosh (7 \theta) \\
v_{2} \cosh (7 \theta) & \cosh (7 \theta) & \cosh (7 \theta)-v_{3} \sinh (7 \theta) & \left(v_{1}-v_{2}\right) \cosh (7 \theta) \\
v_{3} \cosh (7 \theta) & -v_{2} \cosh (7 \theta) & v_{1} \cosh (7 \theta) & \sinh (7 \theta)
\end{array}\right),
$$

where $v_{1}=v_{2}=v_{3}=\frac{1}{\sqrt{2}}$ and $\theta=\arcsin h(1)$. If we calculate the 7 th root of $Z_{R}$, we get

$$
X_{0}=\left(\begin{array}{cccc}
\sinh (\beta) & \left(v_{2}-v_{1}\right) \cosh (\beta) & v_{1} \cosh (\beta) & v_{3} \cosh (\beta) \\
v_{1} \cosh (\beta) & \sinh (\beta)+v_{3} \cosh (\beta) & 0 & -v_{1} \cosh (\beta) \\
v_{2} \cosh (\beta) & v_{3} \cosh (\beta) & \sinh (\beta)-v_{3} \cosh (\beta) & \left(v_{1}-v_{2}\right) \cosh (\beta) \\
v_{3} \cosh (\beta) & -v_{2} \cosh (\beta) & v_{1} \cosh (\beta) & \sinh (\beta)
\end{array}\right) \text {, }
$$

where $\beta=\frac{\theta}{7}$. Its numerical value is given as

$$
X_{0}=\left(\begin{array}{cccc}
0.126 & 0 . & 0.712 & 0.712 \\
0.712 & 0.838 & 0 . & -0.712 \\
0.712 & 1.414 & 1.325 & 0 . \\
0.712 & -0.712 & 0.712 & 0.126
\end{array}\right)
$$

Example 4. Let us consider the hybrid number as $Z=1+i+h$. Since $C(Z)=1$ and $C_{\varepsilon}(Z)=0$, then this hybrid number is called unit timelike parabolic hybrid number. Moreover, the polar form of $Z$

$$
Z=1\left(1+V_{0}\right),
$$

where $V_{0}=i+h$. The matrix representation of $Z$ is given as follows:

$$
Z_{R}=\left(\begin{array}{cccc}
1 & -1 & 1 & 1 \\
1 & 2 & 0 & -1 \\
0 & 1 & 0 & -1 \\
1 & 0 & 1 & 1
\end{array}\right)
$$

For $n=3$,

$$
Z_{R}^{3}=\left(\begin{array}{cccc}
1 & -3 & 3 & 3 \\
3 & 4 & 0 & -3 \\
0 & 3 & -2 & -3 \\
3 & 0 & 3 & 1
\end{array}\right)
$$

If we calculate the 3 th root of $\mathbf{Z}_{R}$, we get

$$
X_{0}=\left(\begin{array}{cccc}
1 & -\frac{1}{3} & \frac{1}{3} & \frac{1}{3} \\
\frac{1}{3} & \frac{4}{3} & 0 & -\frac{1}{3} \\
0 & \frac{1}{3} & \frac{2}{3} & -\frac{1}{3} \\
\frac{1}{3} & 0 & \frac{1}{3} & 1
\end{array}\right)
$$


Example 5. Let us consider the hybrid number as $Z=-1-\varepsilon+h$. Since $C(Z)=0$ and $C_{\varepsilon}(Z)=1$, this hybrid number is called unit lightlike hyperbolic hybrid number. Moreover, the polar form of $Z$

$$
Z=-1\left(1+V_{0}\right)
$$

where $V_{0}=\varepsilon-h$. The matrix representation of $\mathrm{Z}$ is given as follows:

$$
Z_{R}=\left(\begin{array}{cccc}
-1 & -1 & 0 & 1 \\
0 & 0 & 0 & 0 \\
-1 & 1 & -2 & -1 \\
1 & 1 & 0 & -1
\end{array}\right)
$$

For $n=3$,

$$
Z_{R}^{3}=\left(\begin{array}{cccc}
-4 & -4 & 0 & 4 \\
0 & 0 & 0 & 0 \\
-4 & 4 & -8 & -4 \\
4 & 4 & 0 & -4
\end{array}\right) .
$$

If we calculate the 3 th root of $\mathbf{Z}_{R}$, we get

$$
X_{0}=\left(\begin{array}{cccc}
\frac{\sqrt[3]{-2}}{2} & \frac{\sqrt[3]{-2}}{2} & 0 & -\frac{\sqrt[3]{-2}}{2} \\
0 & 0 & 0 & 0 \\
\frac{\sqrt[3]{-2}}{2} & -\frac{\sqrt[3]{-2}}{2} & \sqrt[3]{-2} & \frac{\sqrt[3]{-2}}{2} \\
-\frac{\sqrt[3]{-2}}{2} & -\frac{\sqrt[3]{-2}}{2} & 0 & \frac{\sqrt[3]{-2}}{2}
\end{array}\right) .
$$

\section{Conclusions}

The De Moivre's and Euler formulas have been initially computed for complex numbers. Then many authors studied on computation these formulas for some well-known number sequences and some types of quaternions. Recently, there has been huge interest in a new numeric set, which brings together three numerical systems: complex, hyperbolic and dual numbers, called as hybrid number. Inspiring of the papers in the literature, in this study, we compute De Moivre's and Euler formulas for $4 \times 4$ matrices of hybrid numbers by considering the casual characters and types of hybrid vectors, separately. Moreover, we give some illustrative examples to support the main formulas.

Author Contributions: The authors M.A., S.Y.A., E.K. and F.Y. contributed equally to this work. All authors have read and agreed to the published version of the manuscript.

Funding: This research received no external funding.

Data Availability Statement: Not applicable.

Conflicts of Interest: The authors declare no conflict of interest.

\section{References}

1. Cho, E. Euler's Formula and De Moivre's Formula For Quaternions. Mo. J. Math. Sci. 1999, 11, 2. [CrossRef]

2. Jafari, M.; Mortazaasl, H.; Yayli, Y. De Moivre's Formula for Matrices of Quaternions. J. Algebr. Number Theory Appl. 2011, 21, 57-67.

3. Kabadayi, H.; Yayli, Y. De Moivre's Formula for Dual Quaternions. Kuwait J. Sci. 2011, 38, 15-23.

4. Özdemir, M. The Roots of Split Quaternion. Appl. Math. Lett. 2009, 22, 258-263. [CrossRef]

5. Erdogdu, M.; Ozdemir, M. De Moivre's and Euler Formulas for Matrices of Split Quaternions. 2015. Available online: https: / / arxiv.org/abs/1503.05413.pdf (accessed on 12 July 2021).

6. Jafari, M.; Yayli, Y. Matrix Theory Over the Split Quaternions. Int. J. Geom. 2014, 3, 57-69.

7. Ercan, Z.; Yüce, S. On Properties of the Dual Quaternions. Eur. J. Pure Appl. Math. 2011, 4, 142-146.

8. Kösal, H.H.; Bilgili, T. Euler and De Moivre's Formulas for Fundamental Matrices of Commutative Quaternions. Int. Electron. J. Geom. 2020, 13, 98-107. [CrossRef]

9. Kosal, I.A. A note on hyperbolic quaternions. Univers. J. Math. Appl. 2018, 1, 155-159.

10. Szynal-Liana, A.; Włoch, I. On Special Spacelike Hybrid Numbers. Mathematics 2020, 8, 1671. [CrossRef] 
11. Bród, D.; Szynal-Liana, A.; Włoch, I. Two Generalizations of Dual-Hyperbolic Balancing Numbers. Symmetry 2020, $12,1866$. [CrossRef]

12. Kim, J.E. A Representation of De Moivre's Formula Over Pauli-Quaternions. Ann. Acad. Rom. Sci. Ser. Math. Appl. 2017, 9, 145-151.

13. Ozdemir, M. Introduction to Hybrid Numbers. Adv. Appl. Clifford Algebr. 2018, 28, 11. [CrossRef]

14. Öztürk, İ.; Özdemir, M. Similarity of hybrid numbers. Math. Methods Appl. Sci. 2020, 15, 8867-8881. [CrossRef]

15. Özdemir, M. Finding $n$th Roots of a $2 \times 2$ Real Matrix Using De Moivre's Formula. Adv. Appl. Clifford Algebr. 2019, 29, 1-25. [CrossRef] 